\section{Köhler} B. Schönhofer

\title{
Pneumologie und Beatmungsmedizin
}

\author{
Pulmonology and Mechanical Ventilation
}

Es ist bedauerlich, aber entspricht den Tatsachen, dass die internistische Intensivmedizin in Deutschland wissenschaftlich und in den Medien schlecht aufgestellt ist. So ist auch das Organ der Dachgesellschaft DIVI „Internistische Intensiv- und Notfallmedizin“ in medline nicht vertreten und damit international praktisch nicht existent. Demgegenüber sind zwei anästhesiologische Zeitschriften gelistet.

Zumindest für den Bereich der Beatmungsmedizin ist die Situation jetzt anders. Unser in medline gelistetes Journal Pneumologie wird sich diesem Gebiet in der Zukunft deutlich mehr annehmen, was im Wesentlichen mit der wachsenden Bedeutung der Pneumologie in der Intensivmedizin zu tun hat. Äußerlich sichtbar wird diese Entwicklung durch die Satzungs- und Namensänderung der DGP in „Deutsche Gesellschaft für Pneumologie und Beatmungsmedizin“, die jetzt endlich im Vereinsregister eingetragen und damit gültig wurde.

Dieser zusätzliche Schwerpunkt unseres Fachgebietes kommt in der Serie „Beatmung“, die mit der aktuellen Ausgabe der Pneumologie beginnt, zum Ausdruck. In Übersichtsarbeiten wird das gesamte Spektrum der Beatmungsmedizin behandelt. Die einzelnen Kapitel wurden von Experten verfasst, die alle vor Ort auf Intensivstationen tätig sind und ihre praktische Erfahrung in den Artikeln zum Ausdruck bringen. Das ist sehr wichtig, denn gerade in der Intensivmedizin macht die Wichtung der Literaturauswahl durch die persönliche Erfahrung des Autoren die Qualität und praktische Relevanz einer Übersichtsarbeit aus.
Die Serie beginnt bei den pathophysiologischen Grundlagen, weiter werden die Beatmung bei akuter und chronischer ventilatorischer Insuffizienz sowie bei Hypoxie und Linksherzinsuffizienz und die Strategien bei Weaning dargestellt. Ebenso wird das technische Equipment mit den Beatmungsmodi und der Logistik der Beatmung behandelt. Schließlich werden Aspekte zur Lebensqualität und vor allem zur Ethik am Lebensende angesprochen, die jedem Arzt, der mit diesen schwerkranken Patienten zu tun hat, auf den Nägeln brennen.

In der Intensivmedizin ist die Datenlage für die praktische Arbeit mit den schwerkranken Menschen schwach. Grundlage vieler Therapiekonzepte ist die Erfahrung. Allerdings hat sich die Situation gerade in der Beatmungsmedizin in den vergangenen Jahren deutlich gebessert. Beim hyperkapnischen Atmungsversagen infolge exazerbierter COPD gibt es ausgezeichnete randomisierte kontrollierte Studien, die einen erheblichen Vorteil für die nicht invasive Beatmung gegenüber der konventionellen Beatmung mit Intubation zeigen. Trotzdem bleibt vieles offen und wir würden uns freuen, wenn die Serie als Diskussionsforum genutzt würde. Wir möchten Sie auch stimulieren, Ihre eigene Erfahrung in Leserbriefen einzubringen und mit den Autoren zu diskutieren.

Diese Serie soll auch dazu anregen, vor allem im Bereich der Beatmungsmedizin vermehrt wissenschaftlich zu arbeiten. Hier ist ein großer Nachholbedarf und gerade die Pneumologen mit ihrem pathophysiologischem Verständnis des Gasaustausches und der Lungenmechanik können viel hierzu beitragen. 